\title{
Solution of One -dimensional Fractional Diffusion Equations Involving Caputo Fractional Derivatives in terms of the Mittag - Leffler Functions
}

\author{
R.K. Saxena \\ Department of Mathematics and Statistics, Jai Narain Vyas University, Jodhpur 342004, India \\ *Corresponding author: ram.saxena@yahoo.com (R.K.Saxena)
}

Copyright (C) 2014 Horizon Research Publishing All rights reserved.

\begin{abstract}
The object of this article is to investigate the solutions of one-dimensional linear fractional diffusion equations defined by (2.1) and (4.1). The solutions are obtained in a closed and elegant forms in terms of the $\mathrm{H}$-function and generalized Mittag - Leffler functions, which are suitable for numerical computation. The derived results include the results for the one-dimentional linear fractional telegraph equation due to Orsingher and Beghin [1], and recently derived results by Saxena ,Mathai and Haubold [2].
\end{abstract}

Keywords Fractional Diffusion equation, Laplace transform, Fourier transform, Generalized Mittag-Leffler function, $\mathrm{H}$-function, Caputo fractional derivative

\section{Introduction}

Reaction diffusion models have found numerous applications in pattern formation in biology, chemistry, and physics, see, Murray [3] and Cross and Hohenberg [4]. These systems show that diffusion can produce the spontaneous formation of spatio- temporal patterns. For details, interested reader can refer to the work of Turing [5]. A general model for reaction -diffusion systems is discussed by Henry and Wearne [6]. A piecewise linear approach in connection with the diffusive processes has been developed by Strier .Zanette and Wio [7], which leads to the analytic results in reaction - diffusion systems of wave fronts in a bistable reaction -diffusion system with density- dependent diffusivity.

The simplest reaction - diffusion models can be described by an equation of the form

$$
\frac{\partial N}{\partial t}=D \frac{\partial^{2} N}{\partial x^{2}}+F(N), \mathrm{N}=\mathrm{N}(\mathrm{x}, \mathrm{t}),
$$

where $\mathrm{D}$ is the diffusion constant and $\mathrm{F}(\mathrm{N})$ is a nonlinear function representing reaction

kinetics. It is interesting to observe that for
$F(N)=\gamma N(1-N), \quad(1.1) \quad$ reduces $\quad$ to FisherKolmogorov equation and if , however, we set $F(N)=\gamma N\left(1-N^{2}\right)$, it reduces to the real GinsburgLandau equation.

During the last two decades. interest is developed in formulating fractional diffusion equations due to their importance and usefulness in numerous problems arising in certain areas of science . engineering and technology. In this connection, one can refer to the works of Gorenflo ,Iskende rov and Luchko [8], Hilfer [9], Mainardi, Luchko and Pagnini [10],Mainardi ,Luchko and Pagnini [10], Mainardi, Pagnini and Saxena [11], Saichev and Zaslavasky [12],Metzler and Klafter [13], Metzler and Nonnenmacher [14], Anh and Leonenko [15], Haubold ,Mathai and Saxena [16] and others.

A detailed and comprehensive account of fractional differential equations is available from the monographs written by Podlubny [17] and Kilbas ,Srivastava and Trujillo [18] and a survey paper by Metzler and Klafter [13].

In this article, we present the solutions of one-dimensional linear fractional diffusion equations defined by (2.1) and (4.1). The results are derived in a closed form by the application of Laplace and Fourier transforms in terms of the $\mathrm{H}$-functions and generalized Mittag-Leffler functions, which are suitable for numerical computation. Some interesting special cases of the derived results are also given. The present study is in continuation of our investigations reported earlier in the papers by Saxena,Mathai and Haubold $[19.20 .21,22,23,24]$.

\section{Solution of One-dimensional Linear Fractional Diffusion Equation}

In this section, it is proposed to derive the solution of one-dimensional linear fractional diffusion equation (2.1) associated with three Caputo derivatives of fractional orders.

In what follows, the symbol " " will stand for the Laplace transform and "* " for the Fourier transform. 
The first result is given in the form of the following Theorem 1. Consider the one-dimensional linear fractional diffusion equation in the form

$$
\begin{gathered}
{ }_{0} D_{t}^{\alpha} N(x, t)+a_{0} D_{t}^{\beta} N(x, t)+b_{0} D_{t}^{\gamma} N(x, t)=v^{2}{ }_{-\infty} D_{x}^{\eta} N(x, t), \\
(0<\alpha \leq 1,0<\beta \leq 1,0<\gamma \leq 1, \eta>0)
\end{gathered}
$$

with initial conditions

$$
\mathrm{N}(\mathrm{x}, 0)=\mathrm{f}(\mathrm{x}), \quad x \in \mathfrak{R}, \lim _{x \rightarrow \pm \infty} N(x, t)=0, t>0,
$$

where $v^{2} \in R, v^{2} \neq 0$, is a diffusion constant, are the Caputo fractional derivatives of order $\alpha>0, \beta>0, \gamma>0$, respectively and , $a, b \in R_{+\cdot}{ }_{0} D_{t}^{\alpha},{ }_{0} D_{t}^{\beta},{ }_{0} D_{t}^{\gamma}$ are defined by (6.13), ${ }_{-\infty} D_{x}^{\eta}$ is the Liouville partial fractional derivative of order $\eta>0$, defined by (6.15), and $\mathrm{f}(\mathrm{x})$ is a prescribed function, then under the above conditions, there holds the following formula for the solution of (2.1):

$$
\begin{gathered}
N(x, t)=\frac{1}{2 \pi} \sum_{r=0}^{\infty}(-1)^{r} \int_{-\infty}^{\infty} e^{-i k x} \sum_{\ell=0}^{r}\left(\begin{array}{l}
r \\
\ell
\end{array}\right) a^{\ell} b^{r-\ell} t^{(\alpha-\gamma) r+(\gamma-\beta) \ell} f^{*}(k)\left\{E_{\alpha,(\alpha-\gamma) r+(\gamma-\beta) \ell+1}^{r+1}\left(-c t^{\alpha}\right)\right. \\
\left.+a t^{\alpha-\beta} E_{\alpha,(\alpha-\gamma) r+(\gamma-\beta) \ell+1}^{r+1}\left(-c t^{\alpha}\right)+b t^{\alpha-\gamma} E_{\alpha,(\alpha-\gamma) r+(\gamma-\beta) \ell+1}^{r+1}\left(-c t^{\alpha}\right)\right\} d k
\end{gathered}
$$

where $c=v^{2}|k|^{\eta}$ and provided that the series and integrals in (2.4) are convergent.

Proof. Applying the Laplace transform with respect to the time variable $t$ and (6.14) and using the boundary conditions, we find that

$$
{ }_{s}^{\alpha} N^{\sim}(x, s)-s^{\alpha-1} f(x)+a s^{\beta} N^{\sim}(x, s)-a s{ }^{\beta-1} f(x)+b s^{\gamma} N^{\sim}(x, s)-b s^{\gamma-1} f(x) . \quad=v^{2}{ }_{-\infty} D_{x}^{\eta} N^{\sim}(x, s)
$$

If we apply the Fourier transform with respect to the space variable $x$ and apply the results (6.19), (6.20) and (6.21), it yields

$$
\begin{gathered}
s^{\alpha} N^{\sim^{*}}(k, s)-s^{\alpha-1} f^{*}(k)+a s^{\beta} N^{\sim^{*}}(k, s)-a s^{\beta-1} f^{*}(k)+b s^{\gamma} N^{\sim^{*}}(k . s)-b s^{\gamma-1} f^{*} \\
=-v^{2}|k|^{\eta} N^{\sim^{*}}(k, s)
\end{gathered}
$$

Solving for $N^{\sim^{*}}(k, s)$, it gives

$$
N^{\sim^{*}}(k, s)=\frac{\left(s^{\alpha-1}+a s^{\beta-1}+b s^{\gamma-1}\right) f^{*}(k)}{s^{\alpha}+a s^{\beta}+b s^{\gamma}+c},
$$

where $c=v^{2}|k|^{\eta}$.

To invert the equation (2.7), it is convenient to first invert the Laplace transform and then the Fourier transform. Inverting the Laplace transform with the help of the results (6.19),(6.20) and (6.21), it yields

$$
\begin{aligned}
N^{*}(k, t)= & \sum_{r=0}^{\infty}(-1)^{r} \sum_{\ell=0}^{r}\left(\begin{array}{l}
r \\
\ell
\end{array}\right) a^{\ell} b^{r-\ell} t^{(\alpha-\gamma) r+(\gamma-\beta) \ell} f^{*}(k){ }_{\left[E_{\alpha,(\alpha-\gamma) r+(\gamma-\beta) \ell+1}^{r+1}\left(-c t^{\alpha}\right)\right.} \\
& \left.+a t^{\alpha-\beta} E_{\alpha,(\alpha-\gamma) r+(\gamma-\beta) \ell+\alpha-\beta+1}^{r+1}\left(-c t^{\alpha}\right)+b t^{\alpha-\gamma} E_{\alpha,(\alpha-\gamma) r+(\gamma-\beta) \ell+\alpha-\gamma+1}^{r+1}\left(-c t^{\alpha}\right)\right]
\end{aligned}
$$

Finally, the inverse Fourier transform of $(2,8)$ gives the desired solution in the form

$$
\begin{aligned}
N(x, t)= & \frac{1}{2 \pi} \sum_{r=0}^{\infty}(-1)^{r} \int_{-\infty}^{\infty} e^{-i k x} \sum_{\ell=0}^{r}\left(\begin{array}{l}
r \\
\ell
\end{array}\right) a^{\ell} b^{r-\ell} t^{(\alpha-\gamma) r+(\gamma-\beta) \ell} f^{*}(k) \\
& +a t^{\alpha-\beta} E_{\alpha,(\alpha-\gamma) r+(\gamma-\beta) k+11}^{r+1}{ }^{\left(-c t^{\alpha}\right)+b t^{\alpha-\gamma} E_{\alpha,(\alpha-\gamma) r+(\gamma-\beta) k+1}^{r+1}\left(-c t^{\alpha}\right)}
\end{aligned}
$$


This completes the proof of Theorem 1 .

\section{Special Cases and Fundamental Solutions}

Some special cases of theorem 1 are given below.

(i) If we set $\alpha=\beta=\gamma=\eta=1 / 2$, the theorem reduces to

Corollary 1.1. Consider the one-dimensional linear fractional diffusion equation

$$
{ }_{0} D_{t}^{1 / 2} N(x, t)+a_{0} D_{t}^{1 / 2} N(x, t)+b_{0} D_{t}^{1 / 2} N(x, t)=v^{2}{ }_{-\infty} D_{x}^{1 / 2} N(x, t)
$$

with initial conditions

$$
\mathrm{N}(\mathrm{x}, 0)=\mathrm{f}(\mathrm{x}),, x \in \mathfrak{R}, \lim _{x \rightarrow \pm \infty} N(x, t)=0, t>0,
$$

where $v^{2} \in R, v^{2} \neq 0$, is a diffusion constant, $\mathrm{f}(\mathrm{x})$ is a prescribed function, then there holds the following formula for the solution of (3.1):

$$
N(x, t)=\frac{(1+a+b)}{2 \pi} \sum_{r=0}^{\infty}(-1)^{r} \int_{-\infty}^{\infty} e^{-i k x} \sum_{\ell=0}^{r}\left(\begin{array}{l}
r \\
\ell
\end{array}\right) a^{\ell} b^{r-\ell} f^{*}(k) E_{1 / 2,1}^{r+1}\left(-\zeta t^{\alpha}\right) d k
$$

where $\zeta=v^{2}|k|^{1 / 2}$.

(ii) When $f(x)=\delta(x)$, where $\delta(x)$ is the Dirac -delta function, the theorem yields the following result

Corollary 1.2. Consider the one-dimensional linear fractional diffusion equation

$$
\begin{gathered}
{ }_{0} D_{t}^{\alpha} N(x, t)+a_{0} D_{t}^{\beta} N(x, t)+b_{0} D_{t}^{\gamma} N(x, t)=v^{2}{ }_{-\infty} D_{x}^{\eta} N(x, t) \\
(0<\alpha \leq 1,0<\beta \leq 1,0<\gamma \leq 1, \eta>0)
\end{gathered}
$$

subject to the initial conditions

$$
\mathrm{N}(\mathrm{x}, 0)=\delta(x), x \in \mathfrak{R}, \lim _{x \rightarrow \pm \infty} N(x, t)=0, t>0,
$$

where $v^{2} \in R, v^{2} \neq 0$, is a diffusion constant, $a, b \in R_{+, 0} D_{t}^{\alpha},{ }_{, 0} D_{t}^{\beta},,_{0} D_{t}^{\gamma}$ are the Caputo fractional derivatives of order $\alpha>0, \beta>0, \gamma>0$, respectively and defined by (A.13), ${ }_{-\infty} D_{x}^{\eta}$ is the Liouville partial fractional derivative of order $\eta>0$ and $\delta(x)$ is a Dirac-delta function, then for the fundamental solution of (3.4) under the given initial conditions,, there holds the formula

$$
\begin{aligned}
& N(x, t)=\frac{1}{2 \pi} \sum_{r=0}^{\infty}(-1)^{r} \int_{-\infty}^{\infty} e^{-i k x} \sum_{\ell=0}^{r}\left(\begin{array}{l}
r \\
\ell
\end{array}\right) a^{\ell} b^{r-\ell} t^{(\alpha-\gamma) r+(\gamma-\beta) \ell}\left\{E_{\alpha,(\alpha-\gamma) r+(\gamma-\beta) \ell+1}^{r+1}\left(-c t^{\alpha}\right)\right. \\
&\left.+a t^{\alpha-\beta} E_{\alpha,(\alpha-\gamma) r+(\gamma-\beta) k+11}^{r+1}\left(-c t^{\alpha}\right)+b t^{\alpha-\gamma} E_{\alpha,(\alpha-\gamma) r+(\gamma-\beta) \ell+1}^{r+1}\left(-c t^{\alpha}\right)\right\} d k
\end{aligned}
$$

where $c=v^{2}|k|^{\eta}$, and provided that the series and integrals in (3.7) are convergent.

As $b \rightarrow 0$, then making slight changes in the parameters, we obtain the following result obtained by Saxena, Mathai and Haubold [2]:

Corollary 1.3. Consider the one-dimensional linear fractional diffusion equation

$$
\begin{gathered}
{ }_{0} D_{t}^{\alpha} N(x, t)+a_{0} D_{t}^{\beta} N(x, t)=v^{2}{ }_{-\infty} D_{x}^{\gamma} N(x, t), \\
(0<\alpha \leq 1,0<\beta \leq 1, \gamma>0)
\end{gathered}
$$

with initial conditions

$$
\mathrm{N}(\mathrm{x}, 0)=\mathrm{f}(\mathrm{x}), x \in \mathfrak{R}, \lim _{x \rightarrow \pm \infty} N(x, t)=0, t>0,
$$


where $v^{2} \in R, v^{2} \neq 0$, is a diffusion constant, $a \in R_{+}, 0 D_{t}^{\alpha},{ }_{, 0} D_{t}^{\beta}$, are the Caputo fractional derivatives of order $\alpha>0, \beta>0$ respectively and defined by (6.13), ${ }_{-\infty} D_{x}^{\gamma}$ is the Liouville partial fractional derivative of order $\gamma>0$, defined by $(6.15)$ and $\mathrm{f}(\mathrm{x})$ is a prescribed function, then there holds the following formula for the solution of (3.8):

$$
\begin{gathered}
N(x, t) \\
=\sum_{r=0}^{\infty} \frac{(-1)^{r}}{2 \pi} \int_{-\infty}^{\infty} t^{(\alpha-\beta) r} f^{*}(k) \exp (-k x)\left[E_{\alpha,(\alpha-\beta) r+1}^{r+1}\left(-d t^{\alpha}\right)+t^{\alpha-\beta} E_{\alpha,(\alpha-\beta)(r+1)+1}^{r+1}\left(-d t^{\alpha}\right)\right] d k
\end{gathered}
$$

where $\alpha>\beta$ and

$$
d=v^{2}|k|^{\gamma} .
$$

Now if we set $f(x)=\delta(x), \mathrm{b}=0, \eta=2, \alpha$ is replaced by $2 \alpha$ and $\beta$ by $\alpha$ in (3.8) and use the result (6.25), we then obtain the following interesting result, which includes many known results on fractional telegraph equations including the one recently given by Orsingher and Beghin [1].

Corollary 1.4. Consider the one-dimensional linear fractional diffusion equation

$$
\frac{\partial^{2 \alpha} N(x, t)}{\partial t^{2 \alpha}}+a \frac{\partial^{\alpha} N(x, t)}{\partial t^{\alpha}}=v^{2} \frac{\partial^{2} N(x, t)}{\partial x^{2}}, 0<\alpha \leq 2
$$

with the initial conditions

$$
N(x, 0)=\delta(x), x \in \mathfrak{R}, N_{t}(x, 0)=0, \lim _{x \rightarrow \pm \infty} N(x, t)=0, t>0,
$$

where $v^{2} \in R, v^{2} \neq 0$, is a diffusion constant, $a \in R_{+}, \delta(x)$ is a Dirac-delta function, then for the fundamental solution of (3.12) under the above constraints, there holds the following formula :

$$
N(x, t)=\frac{1}{2 \pi \sqrt{\left(a^{2}-4 b\right)}} \int_{-\infty}^{+\infty} \exp (-i k x)\left\{(\lambda+a) E_{\alpha}\left(\lambda t^{\alpha}\right)-(\mu+a) E_{\alpha}\left(\mu t t^{\alpha}\right)\right\} d k
$$

where $\lambda$ and $\mu$ are the real and distinct roots of the quadratic equation

$$
y^{2}+a y+b=0
$$

given by

$$
\lambda=\frac{1}{2}\left(-a+\sqrt{\left(a^{2}-4 b\right)}\right) \text { and } \mu=\frac{1}{2}\left(-a-\sqrt{\left(a^{2}-4 b\right)},\right.
$$

where $b^{2}=v^{2} k^{2}, E_{\alpha}(x)$ is the Mittag - Leffler function defined by (6.1).and provided that the integral appearing in (3.14) is convergent.

The equation (3.14) represents the solution of time-fractional telegraph equation (3.12) subject to the initial conditions (3.13), recently solved by Orsingher and Beghin [1]. It may be remarked here that the solution as given by Orsingher et al [1] is in terms of the Fourier transform of the solution in the form given below.

The Fourier transform of the solution (3.14) of the equation (3.12) can be expressed in the form

$$
N^{*}(x, t)=\frac{1}{2}\left\{\left(1+\frac{a}{\sqrt{\left(a^{2}-4 v^{2} k^{2}\right)}}\right) E_{\alpha}\left(\lambda t^{\alpha}\right)+\left(1-\frac{a}{\sqrt{\left(a^{2}-4 v^{2} k^{2}\right)}}\right) E_{\alpha}\left(\mu t^{\alpha}\right)\right\},
$$

where $\lambda$ and $\mu$ are defined in (3.16) and $E_{\alpha}(x)$ is the Mittag -Leffler function.

Following a similar procedure, the following general theorem, which provides an extension of Theorem 1. can be established, which makes use of the result (6.22). 


\section{Extension of Theorem 1}

In this section, we present a generalization of Theorem 1 proved in section 2, which can be established in a similar manner by using the result (6.22).

Theorem 2. Consider the one-dimensional linear fractional diffusion equation associated with a finite number of Caputo derivatives of fractional orders in the following form:

$$
\begin{aligned}
& a_{1} D_{t}^{\alpha 1} N(x, t)+a_{2} D_{t}^{\alpha 2} N(x, t)+\ldots+a_{n+1} D_{t}^{\alpha}{ }_{n+1} N(x, t)=v^{2}{ }_{-\infty} D_{x}^{\mu} N(x, t, \\
& 0<\alpha_{j} \leq 1,(j=1, \ldots, n+1), \mu>0
\end{aligned}
$$

with initial conditions

$$
\mathrm{N}(\mathrm{x}, 0)=\mathrm{f}(\mathrm{x}), \quad x \in \mathfrak{R}, \lim _{x \rightarrow \pm \infty} N(x, t)=0, t>0,
$$

where $v^{2} \in R, v^{2} \neq 0$, is a diffusion constant, $a_{j} \in R_{+} ; 0 D_{t}^{\alpha} j(\mathrm{j}=1, \ldots, \mathrm{n}+1)$ are the Caputo fractional derivatives of order $\alpha_{j}>0,(j=1, \ldots, n+1)$, defined by (6.13), ${ }_{-\infty} D_{x}^{\mu}$ is the Liouville partial fractional derivative of order $\mu>0$, defined by (6.15) and $\mathrm{f}(\mathrm{x})$ is a prescribed function, then under the given conditions, there holds the following formula for the solution of (4.1).

$$
\begin{gathered}
N(x, t)=\frac{1}{2 \pi a_{1}} \\
\sum_{m=0}^{\infty}(-1)^{m} \int_{-\infty}^{\infty} f^{*}(k) \exp (-i k x) \sum_{r+\ldots+m=m} \frac{m !}{r_{1} ! \ldots r_{n} !}\left\{\prod_{j=1}^{n}\left(\frac{a_{j+1}}{a_{1}}\right)^{r j}\right\} \\
\times t^{P} \sum_{\omega=1}^{n+1} a_{\omega} t^{-\alpha} \omega_{E_{1}, A-\alpha_{\omega}+1}^{m+1}\left(-\frac{\tau}{a_{1}} t^{\alpha_{1}}\right) d k
\end{gathered}
$$

where $\tau=v^{2}|k|^{\mu}$

$$
\begin{gathered}
\operatorname{Re}\left(\alpha_{j+1}-\alpha_{2}\right)>0 \text { for } \mathrm{j}=2, \ldots, \mathrm{n}, \\
\mathrm{P}=\sum_{j=1}^{n}(\alpha 2-\alpha j+1) r j+\alpha 1(1+m)-\alpha 2^{m}, \\
a_{1} \neq 0
\end{gathered}
$$

and provided that the series and integrals in (4.4) are convergent.

\section{Consequences of General Theorem 2}

When $\alpha_{j}=\mu=1 / 2 \quad(\mathrm{j}=1,2, \ldots, \mathrm{n}+1)$, the Theorem 2 yields

Corollary 2.1. Consider the one-dimensional linear fractional diffusion equation associated with Caputo derivatives in the form

$$
\begin{gathered}
a_{1} D_{t}^{1 / 2} N(x, t)+a_{20} D_{t}^{1 / 2} N(x, t)+\ldots+a_{n+1} D_{t}^{1 / 2} N(x, t)=v^{2}{ }_{-\infty} D_{x}^{1 / 2} N(x, t) \\
\left(0<\alpha_{j} \leq 1,(j=1, \ldots, n+1)\right.
\end{gathered}
$$

with initial conditions 


$$
\mathrm{N}(\mathrm{x}, 0)=\mathrm{f}(\mathrm{x}),(x \in \mathfrak{R}), \lim _{x \rightarrow \pm \infty} N(x, t)=0, t>0,
$$

where $v^{2} \in R, v^{2} \neq 0$, is a diffusion constant, $a_{j} \in R_{+},(j=1, \ldots .,(n+1))$ and $\mathrm{f}(\mathrm{x})$ is a prescribed function, then there holds the following formula for the solution of (5.1):

$$
\begin{gathered}
N(x, t)=\frac{1}{2 \pi a_{1}} \\
\sum_{m=0}^{\infty}(-1)^{m} \int_{-\infty}^{\infty} f^{*}(k) \exp (-i k x) \sum_{1+\ldots+m=m} \frac{m !}{r_{1} ! \ldots . r_{n} !}\left\{\prod_{j=1}^{n}\left(\frac{a_{j+1}}{a_{1}}\right)^{r j}\right\} \times\left(a_{1}+\ldots+a_{n+1}\right) E_{1 / 2,1}^{m+1}\left(-\frac{\zeta t^{1 / 2}}{a_{1}}\right) d k
\end{gathered}
$$

where $\zeta=v^{2}|k|^{1 / 2}, a_{1} \neq 0$ and provided that the series and integrals in (5.4) are convergent.

On the other hand, if we set $f(x)=\delta(x)$, where $\delta(x)$ is thr Dirac-delta function and use the result (A.12), we arrive at Corollary 2.2. Consider the one-dimensional linear fractional diffusion equation

$$
\begin{gathered}
a_{1} D_{t}^{\alpha 1} N(x, t)+a_{2} D_{t}^{\alpha 2} N(x, t)+\ldots+a_{n+1} D_{t}^{\alpha_{n+1}} N(x, t)=v^{2}{ }_{-\infty} D_{x}^{\mu} N(x, t) \\
\left(0<\alpha_{j} \leq 1,(j=1, \ldots .,(n+1)), \mu>o .\right. \\
N(x, 0)=\delta(x), x \in R, \lim _{x \rightarrow \pm \infty} N(x, t)=0, t>0
\end{gathered}
$$

where $v^{2} \in R, v^{2} \neq 0$, is a diffusion constant, $a_{j} \in R_{+}, 0 D_{t}^{\alpha} j(\mathrm{j}=1, \ldots, \mathrm{n}+1)$ are the Caputo fractional derivatives of order $\alpha_{j}>0,(j=1, \ldots, n+1)$, defined by $(6.13),{ }_{-\infty} D_{t}^{\mu}$ is the

Liouville partial fractional derivative of order $\mu>0$, defined by (6.15) and $\delta(x)$ is the Dirac-delta function, then under the above given conditions, there holds the following formula for the fundamental solution of (5.5).

$$
N(x, t)=\frac{1}{2 \pi a_{1}} \sum_{m=0}^{\infty}(-1)^{m} \sum_{\eta_{1}+\ldots+r_{n}=m} \frac{m !}{r_{1} ! \ldots r_{n} !}\left\{\prod_{j=1}^{n}\left(\frac{a_{j+1}}{a_{1}}\right)^{r j}\right\} \sum_{\omega=1}^{n+1} a_{\omega} G(x-\tau, t) f(\tau) d \tau
$$

where

$$
\begin{gathered}
P=\sum_{j=1}^{n}\left(\alpha_{j+1}-\alpha_{2}\right) r_{j}+\alpha_{1}+\left(\alpha_{1}-\alpha_{2}\right) m \\
\alpha_{j}>0,(j=1, \ldots, n+1) ;\left(\alpha_{j+1}-\alpha_{2}\right)>0
\end{gathered},
$$

for $\mathrm{j}=2, \ldots, \mathrm{n}, \quad \tau=v^{2}|k|^{\mu}$, and $a_{1} \neq 0$;

$$
\begin{aligned}
& G(x, t)=\frac{t^{P-\alpha} \omega}{2 \pi} \int_{-\infty}^{\infty} \exp (-i k x) E_{\alpha_{1}, P-\alpha_{\omega}+1}^{m+1}\left(\frac{v^{2}|k|^{\mu} t^{\alpha^{\prime}}}{a_{1}}\right) d k \\
& =\frac{t^{P-\alpha_{\omega}}}{\pi \mu(m !)} \int_{0}^{\infty} \cos (k x) H_{1,2}^{1,1}\left[\frac{v^{2 / k_{|k| t} \alpha_{1} / \mu}}{a_{1}^{1 / \mu}} \mid \begin{array}{l}
(-m, 1 / \mu) \\
(0,1 / \mu),\left(\alpha_{\omega}-P, \frac{\alpha_{1}}{\mu}\right)
\end{array}\right] \text {, }
\end{aligned}
$$




$$
=\frac{t^{P-\alpha_{\omega}}}{\mu(m !)|x|} H_{3,3}^{2,1}\left[\frac{|x| a^{1 / \mu}}{v^{2 / \mu} \alpha_{1} / \mu} \mid \begin{array}{l}
(1,1 / \mu),\left(1+P-\alpha_{\omega}, \alpha_{1} / \mu\right),(1,1 / 2) \\
(1,1),(1+m, 1 / \mu),(1,1 / 2)
\end{array}\right],
$$

provided that the series and integral in (5.9) are convergent.

\section{Discussion}

It is interesting to observe that the method employed for deriving the solution of the equations (2.1) and (4.1) in the space $L F=L\left(R_{+}\right) \times F(R)$ can also be applied in the space $L F^{\prime}=L^{\prime}\left(R_{+}\right) \times F^{\prime}$, where $F^{\prime}=F^{\prime}(r)$ is the space of Fourier transform of generalized functions. As an illustration, we can choose $\mathrm{F}^{\prime}=\mathrm{S}^{\prime}$ or $\mathrm{F}^{\prime}=\mathrm{D}^{\prime}$. The Fourier transforms in $\mathrm{S}^{\prime}$ and $\mathrm{D}^{\prime}$ are introduced by Schwartz and Gelfand and Shilov, respectively. $S^{\prime}$ is the dual of the space S, which is the space of all infinitely differentiable functions which, together with their derivatives approach zero more rapidly than any power of

$1 /|x|$ as $\quad|x| \rightarrow \infty$. [ Gelfand and Shilov 25, p.16 ]. Here D' is the dual of the space D which consists of all smooth functions with compact supports [ Brychkov and Prudnikov,26,p. 3]. For further details, the reader is referred to the monographs written by Gelfand and Shilov [25] and Brychkov and Prudnikov et al [26], if we replace the Laplace and Fourier transforms in (6.8) and (6.10) by the corresponding Laplace and Fourier transform of the generalized functions.

Due to presence of Mittag-Leffler in the solutions, the results obtained in this article can be computed numerically. This will further enhance the utility of derived results in applied problems of science, engineering, technology and related areas.

\section{Conclusion}

In this article solution of fractional diffusion equations associated with Caputo derivatives of fractional orders are investigated. Since the results contain the Mittag -Leffler function, which is gaining importance day by day due its importance and and utility and applications in applied problems of physical sciences and engineering. The results obtained may find applications in industrial problems, such as Glass industry etc.

\section{Acknowledgements}

The author is grateful to Professor H.J.Haubold for his constant encouragement and interest in my research work.

\section{Appendix}

(i) Mittag-Leffler functions and fractional derivatives

A generalization of the Mittag - Leffler function has been given by Mittag - Leffler [27] as

$$
E_{\alpha}(z)=\sum_{n=0}^{\infty} \frac{z^{n}}{\Gamma(n \alpha+1)}, \quad(\alpha \in C, \operatorname{Re}(\alpha)>0)
$$

The above equation was further generalized by Wiman [28] in the form

$$
E_{\alpha, \beta}(z)=\sum_{n=0}^{\infty} \frac{z^{n}}{\Gamma(n \alpha+\beta)},(\alpha, \beta \in C, \operatorname{Re}(\alpha)>0, \operatorname{Re}(\beta)>0) .
$$

The main results of the functions, defined by (6.1) and (6.2) are available in the book written by Erdélyi ,Wagnus, Oberhettinger and Tricomi [29, Section 18.1] and the monographs written by Dzherbashyan [30,31].

Prabhakar [ 32]in the year 1971 introduced a generalization of (6.2) in the form

$$
\begin{aligned}
& E_{\alpha, \beta}^{\gamma}(z)=\sum_{n=0}^{\infty} \frac{(\gamma)_{n} z^{n}}{\Gamma(n \alpha+\beta)(n) !} \\
& (\alpha, \beta \cdot \gamma \in C ; \operatorname{Re}(\alpha), \operatorname{Re}(\beta), \operatorname{Re}(\gamma)>0)
\end{aligned}
$$


where $(\gamma)_{r}$ is the Pochhammer's symbol, defined by

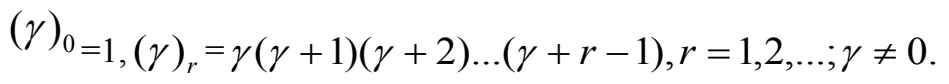

It is an entire function with $\rho=[\operatorname{Re}(\alpha)]^{-1}$,see [Prabhakar,[32]. This function is studied, among others, by Kilbas, Saigo and Saxena $[33,34]$.

For $\gamma=1$, the function (6,3) coincides with (6.2), while for $\beta=\gamma=1$ with (6..1):

$$
E_{\alpha, \beta}^{1}=E_{\alpha, \beta}(z), \quad E_{\alpha, 1}^{1}(z)=E_{\alpha}(z) .
$$

Prabhakar [32, p.8, (2.5)] has shown that

$$
L\left\{t^{\gamma-1} e^{-s t} E_{\beta, \gamma}^{\delta}\left(\omega t^{\beta}\right) ; s\right\}=s^{-\gamma}\left(1-\omega s^{-\beta}\right)^{-\delta}
$$

where $\operatorname{Re}(\beta)>0, \operatorname{Re}(\gamma)>0, \operatorname{Re}(\mathrm{s})>0$ and $s>|\omega|^{\frac{1}{\operatorname{Re}(\beta)}}$.

In terms of the H-function [Mathai ,Saxena and Haubold ,35], it can be expressed in the form

$$
E_{\alpha, \beta}^{\gamma}(-z)=\frac{1}{\Gamma(\gamma)} H_{1,2}^{1,1}\left[\left.z\right|_{(0, \gamma),(1-\beta, \alpha)} ^{(1-, 1)}\right],
$$

where $\operatorname{Re}(\alpha)>0, \operatorname{Re}(\beta)>0, \operatorname{Re}(\gamma)>0$.

The Laplace transform of a function $\mathrm{N}(\mathrm{x}, \mathrm{t})$ with respect to $\mathrm{t}$ is defined by

$$
L\{N(x, t)\}=N^{\sim}(x, s)=\int_{0}^{\infty} e^{-s t} N(x, t) d t,(t>0),(x \in R),
$$

where $\operatorname{Re}(s)>0$, and its inverse transform with respect to $s$ is given by

$$
L^{-1}\left\{N^{\sim}(x, s)\right\}=L^{-1}\left\{N^{\sim}(x, s) ; t\right\}=\frac{1}{2 \pi i} \int_{\gamma-i \infty}^{\gamma+i \infty} e^{s t} N^{\sim}(x, s) d s,
$$

$\gamma$ being a fixed real number.

The Fourier transform of a function $\mathrm{N}(\mathrm{x}, \mathrm{t})$ with respect to $\mathrm{x}$ is defined by

$$
F\{N(x, t)\}=F^{*}(k, t)=\int_{-\infty}^{\infty} e^{i k x} N(x, t) d x .
$$

The inverse Fourier transform with respect to $\mathrm{k}$ is given by the formula

$$
F^{-1}\left\{F^{*}(x, t)\right\}=\frac{1}{2 \pi} \int_{-\infty}^{\infty} e^{-i k x} F^{*}(k, t) d k .
$$

The space of functions for which the transforms defined by (6.8) and (6.10) exist are denoted by $L F=L\left(R_{+}\right) \times F(R)$. The cosine transform of the $\mathrm{H}$-function is given by Metzler and Nonnenmacher, [13]: as

$$
\begin{gathered}
\left.\int_{0}^{\infty} t^{\rho-1} \cos (k t) H_{p, q}^{m, n}\left[a t^{\mu} \mid \begin{array}{c}
\left(a_{p}, A_{p}\right) \\
\left(b_{q}, B_{q}\right)
\end{array}\right)\right] t \\
=\frac{\pi}{k^{\rho}} H_{q+1, p+2}^{n+1, m}\left[\frac{k^{\mu}}{a} \mid \begin{array}{l}
\left(1-b_{q}, B_{q}\right),\left(\frac{1+\rho}{2}, \frac{\mu}{2}\right) \\
(\rho, \mu),\left(1-a_{p}, A_{p}\right),\left(\frac{1+\rho}{2}, \frac{\mu}{2}\right)
\end{array}\right],
\end{gathered}
$$

where $\operatorname{Re}\left[\rho+\mu \operatorname{Re}\left[\rho+\mu_{1 \leq j \leq m}^{\min }\left(\frac{b_{j}}{B_{j}}\right)\right]>1 ; k, \mu>0 ; \operatorname{Re}\left[\rho+\mu_{1 \leq j \leq n}^{\max }\left(\frac{a_{j}-1}{A_{j}}\right)\right]<\frac{3}{2}\right.$. 
$|\arg a|<\frac{1}{2} \pi \theta ; \quad \theta=\sum_{j=1}^{n} A_{j}-\sum_{j=n+1}^{p} A_{j}+\sum_{j=1}^{m} B_{j}-\sum_{j=m+1}^{q} B_{j}>0$

The following fractional derivative of order $\alpha>0$ is introduced by Caputo [36] in the form

$$
\begin{aligned}
{ }_{0} D_{t}^{\alpha} f(x, t) & =\frac{1}{\Gamma(m-\alpha)} \int_{0}^{t} \frac{f^{(m)}(x, \tau) d \tau}{(t-\tau)^{\alpha+1-m}} \quad m-1<\alpha \leq m, \operatorname{Re}(\alpha)>0, m \in N . \\
& =\frac{\partial^{m} f(x, t)}{\partial t^{m}} \quad \text { if } \alpha=m .
\end{aligned}
$$

Where $\frac{\partial^{m} f(x, t)}{\partial t^{m}}$ means the mth partial derivative of $\mathrm{f}(\mathrm{x})$ with respect to $t$.

Caputo [36] has given the Laplace transform of this derivative as

$$
L\left\{{ }_{0} D_{t}^{\alpha} f(x, t)\right\}=s^{\alpha} f^{\sim}(x, s)-\sum_{r=0}^{m-1} s^{\alpha-r-1} f^{(r)}(x, 0+), \quad(m-1<\alpha \leq m)
$$

where $f^{\sim}(x, s)$ is the Laplace transform of $\mathrm{f}(\mathrm{x}, \mathrm{t})$.

The Liouville partial fractional fractional operator given in the monograph by Samko et al,[37,Section 24.2], defined by

$$
{ }_{-\infty} D_{x}^{\mu} f(x, t)=\frac{1}{\Gamma(n-\mu)} \frac{d^{n}}{d t^{n}} \int_{-\infty}^{t} \frac{f(x, u) d u}{(t-u)^{\mu-n+1}}
$$

where $n=[\mu]+1$ is an integral part of $\mu>0$.

The Fourier transform of the above derivative is given by Metzler and Klafter,

$[13$, p.59, A.13]

$$
F\left\{-\infty D_{x}^{\mu} f(x, t)\right\}=-|k|^{\mu} f^{*}(k, t),
$$

Inverse Laplace transform of certain algebraic functions

This section deals with the evaluation of the inverse Laplace transforms of certain algebraic functions which are directly applicable in the analysis that follows.

It will be shown here that

$$
L^{-1}\left\{\frac{s^{\rho-1}}{s^{\alpha}+a s^{\beta}+b s^{\gamma}+c} ; t\right\}=\sum_{r=0}^{\infty}(-1)^{r} \sum_{\ell=0}^{r}\left(\begin{array}{l}
r \\
\ell
\end{array}\right) a^{\ell} b^{r-\ell} t^{(\alpha-\gamma) r+(\gamma-\beta) \ell+\alpha-\rho} E_{\alpha,(\alpha-\gamma) r+(\gamma-\beta) \ell+\alpha-\rho+1}^{r+1}\left(-c t^{\alpha}\right),
$$

Where $(\mathrm{a}, \mathrm{b}, \mathrm{c} \in R)$

$$
\operatorname{Re}(\alpha)>0, \operatorname{Re}(\beta)>0, \operatorname{Re}(\gamma)>0, \operatorname{Re}(s)>0, \operatorname{Re}(\rho)>0,\left|\frac{a s^{\beta}+b s^{\gamma}}{s^{\alpha}+c}\right|<1, \quad E_{\beta, \gamma}^{\delta}(.) \quad \text { is the generalized }
$$

Mittag-Leffler function, defined by (6.3) and provided that the series in (6.17) is convergent.

Proof. Assume that $\alpha>\gamma>\beta$. We have

$$
\begin{aligned}
\frac{s^{\rho-1}}{s^{\alpha}+a s^{\beta}+b s^{\gamma}+c} & =\frac{s^{\rho-\beta-1}}{\left(s^{\alpha-\beta}+c s^{-\beta}\right)\left(1+\frac{a+b s^{\gamma-\beta}}{s^{\alpha-\beta}+c s^{-\beta}}\right)} \\
& =s^{\rho-\beta-1} \sum_{r=0}^{\infty} \frac{(-1)^{r}\left(a+b s^{\gamma-\beta}\right)^{r}}{\left(s^{\alpha-\beta}+c s^{-\beta}\right)^{r+1}} \\
& =\sum_{r=0}^{\infty}(-1)^{r} \sum_{\ell=0}^{r}\left(\begin{array}{l}
r \\
\ell
\end{array}\right) a^{\ell} b^{r-\ell} \frac{s^{\rho+(\gamma-\alpha) r+(\beta-\gamma) \ell-\alpha-1}}{\left(1+c s^{-\alpha}\right)^{r+1}}
\end{aligned}
$$

On term by term inverting (6.18) with the help of the formula (6.6), it readily gives the desired result. The term by term 
inversion is justified by virtue of a theorem due to Doetsch [38, § 22].

Special cases of (6.17). If we set $\rho=\alpha$ in (6.17),we obtain:

$$
\begin{aligned}
& L^{-1}\left\{\frac{s^{\alpha-1}}{s^{\alpha}+a s^{\beta}+b s^{\gamma}+c} ; t\right\} \\
& =\sum_{r=0}^{\infty}(-1)^{r} \sum_{\ell=0}^{r}\left(\begin{array}{l}
r \\
\ell
\end{array}\right) a^{\ell} b^{r-\ell} t^{(\alpha-\gamma) r+(\gamma-\beta) \ell} E_{\alpha,(\alpha-\gamma) r+(\gamma-\beta) \ell+1}^{r+1}\left(-c t^{\alpha}\right)
\end{aligned},
$$

where $\operatorname{Re}(\alpha)>0, \operatorname{Re}(\beta)>0, \operatorname{Re}(\gamma)>0, \operatorname{Re}(s)>0,\left|\frac{a s^{\beta}+b s^{\gamma}}{s^{\alpha}+c}\right|<1$, and provided that the series in (6.19) is convergent.

Similarly, for $\rho=\beta$ in (6.17), it gives

$$
\begin{aligned}
& L^{-1}\left\{\frac{t^{\beta-1}}{s^{\alpha}+a s^{\beta}+b s^{\gamma}+c} ; t\right\} \\
& =\sum_{r=0}^{\infty}(-1)^{r} \sum_{\ell=0}^{r}\left(\begin{array}{l}
r \\
\ell
\end{array}\right) a^{\ell} b^{r-\ell} t^{(\alpha-\gamma) r+(\gamma-\beta) \ell+\alpha-\beta} E_{\alpha,(\alpha-\gamma) r+(\gamma-\beta) \ell+\alpha-\beta+1}^{r+1}\left(-c t^{\alpha}\right)
\end{aligned}
$$

where $\operatorname{Re}(\alpha)>0, \operatorname{Re}(\beta)>0, \operatorname{Re}(\gamma)>0, \operatorname{Re}(s)>0,\left|\frac{a s^{\beta}+b s^{\gamma}}{s^{\alpha}+c}\right|<1$, and provided that the series in (6.20) is convergent.

When $\rho=\gamma$, , 6.17) readily gives the formula

$$
\begin{aligned}
& L^{-1}\left\{\frac{t^{\gamma-1}}{s^{\alpha}+a s^{\beta}+b s^{\gamma}+c} ; t\right\} \\
& =\sum_{r=0}^{\infty}(-1)^{r} \sum_{\ell=0}^{r}\left(\begin{array}{l}
r \\
\ell
\end{array}\right) a^{\ell} b^{r-\ell} t^{(\alpha-\gamma) r+(\gamma-\beta) \ell+\alpha-\gamma} E_{\alpha,(\alpha-\gamma) r+(\gamma-\beta) \ell+\alpha-\gamma+1}^{r+1}\left(-c t^{\alpha}\right)
\end{aligned}
$$

where $\operatorname{Re}(\alpha)>0, \operatorname{Re}(\beta)>0, \operatorname{Re}(\gamma)>0, \operatorname{Re}(s)>0,\left|\frac{a s^{\beta}+b s^{\gamma}}{s^{\alpha}+c}\right|<1$ and provided that the series in (6.21) is convergent.

A general case.

In this section, it will be shown that if $\alpha_{j}>0 \quad a_{j} \in R(\mathrm{j}=1, \ldots, \mathrm{n}), \operatorname{Re}(\mathrm{s})>0$, then

$$
\begin{gathered}
L^{-1}\left[\frac{s^{\rho-1}}{a_{0}+a_{1} s^{\alpha_{1}}+a_{2} s^{\alpha_{2}}+a_{n-2} s^{\alpha_{3}}+\ldots+a_{n} s^{\alpha_{n}}+a_{n+1} s^{\alpha_{n+1}}} ; t\right] \\
\frac{1}{a_{1}} \sum_{m=0}^{\infty}(-1)^{m} \sum_{\substack{r_{1}+r_{2}+\ldots+r_{n}=m \\
\eta \geq 0 \ldots r_{n} \geq 0}} \frac{m !}{r_{1} ! \ldots r_{n} !}\left\{\prod_{j=1}^{n}\left(\frac{a_{j+1}}{a_{1}}\right)^{r j}\right\} t t^{P-\rho_{E^{\prime}}} E_{\alpha_{1}, A-\rho+1}^{m+1}\left(-\frac{a_{0}}{a_{1}} t^{\alpha_{1}}\right), a_{1} \neq 0,
\end{gathered}
$$

where $\mathrm{P}=\sum_{j=1}^{n}(\alpha 2-\alpha j+1) r j+\alpha 1(1+m)-\alpha 2^{m}$

$$
\left|\frac{\sum_{r=1}^{n}\left\{\left(a_{r+1} / a_{1}\right) s^{\alpha_{r+1}-\alpha_{2}}\right\}}{\left(s^{\alpha_{1}-\alpha_{2}}+\left(a_{0} / a_{1}\right) s^{-\alpha_{2}}\right)}\right|<1
$$




$$
a_{1} \neq 0
$$

and provided that the series in (6.22) is convergent.

Proof: Let us assume that $\alpha_{2}>\alpha_{j+1},(j=2,3, \ldots, n)$. We have

$$
\begin{gathered}
\frac{a_{0}+a_{1} s^{\alpha_{1}}+a_{2} s^{\alpha_{2}}+a_{3} s^{\alpha_{3}}+\ldots+a_{n} s^{\alpha_{n}}+a_{n+1} s^{n+1}}{s^{\alpha_{1}-\alpha_{2}}+\left(a_{0} / a_{1}\right) s^{-\alpha_{2}}+\left(a_{2} / a_{1}\right)+\left(a_{3} / a_{1}\right) s^{\alpha_{3}-\alpha_{2}}+\ldots+\left(a_{n} / a_{1}\right) s^{\alpha_{n}-\alpha_{2}}+\left(a_{n+1} / a_{1}\right) s^{\alpha_{n+1}-\alpha_{2}}} \\
=\frac{a_{1}^{-1} s^{\rho-\alpha 2-1}}{\left[s^{\alpha-\alpha}-\left(a_{0} / a_{1}\right) s^{-\alpha 2}\right]\left[1+\frac{\sum_{r=1}^{n}\left\{\left(a_{r+1} / a_{1}\right) s^{\alpha r+1-\alpha 2}\right\}}{\left(s^{\alpha 1-\alpha 2}+\left(a_{0} / a_{1}\right) s^{-\alpha 2}\right)}\right]} \\
=a_{1}^{-1} s^{\rho-\alpha 2-1} \sum_{m=0}^{\infty}(-1)^{m} \frac{\left[\sum_{r=1}^{n}\left\{\left(a_{r+1} / a_{1}\right) s^{\alpha r+1-\alpha 2}\right\}\right]^{m}}{\left(s^{\alpha 1-\alpha 2}+\left(a_{0} / a_{1}\right) s^{-\alpha 2}\right)^{m+1}}
\end{gathered}
$$

On applying the multinomial theorem, [Abramowitz and Stegun,39,p.823;

§24.1.2]:

$$
\left(x_{1}+x_{2}+\ldots+x_{m}\right)^{n}=\sum\left(n ; n_{1}, n_{2}, \ldots, n_{m}\right) x_{1}^{n_{1}} x_{2}^{n_{2}} \ldots x_{m}^{n_{m}} .
$$

summed over $n_{1}+n_{2}+\ldots+n_{m}=n$; the above line transforms into the form

$$
=\frac{1}{a_{1}} \sum_{m=0}^{\infty}(-1)^{m} \sum_{\substack{\eta_{1}+\ldots+r_{n}=m \\ \eta_{1} \geq 0, \ldots, r_{n} \geq 0}} \frac{m !}{r_{1} ! \ldots r_{n} !}\left\{\prod_{j=1}^{n}\left(\frac{a_{j+1}}{a_{1}}\right)^{r j}\right\} \frac{\sum_{j=1}^{n}(\alpha j+1-\alpha 2) r j+\rho+\alpha 2 m-(1+m) \alpha 1-1}{\left(1+\left(a_{0} / a_{1}\right) s^{-\alpha 1}\right)^{m+1}},
$$

Interpreting the above expression with the help of the formula (6.6), we obtain the desired result (6.22). This completes the proof of (6.22).

Finally, from Mathai , Saxenaand Haubold [35,p.186], we have

$$
L^{-1}\left[\frac{s^{\alpha-1}}{a+s^{\beta}} ; t\right]=t^{\beta-\alpha} E_{\beta, \beta-\alpha+1}\left(-a t^{\beta}\right),
$$

where $\alpha, \beta \in C ; \operatorname{Re}(s)>0, \operatorname{Re}(\beta)>0, \operatorname{Re}(\beta-\alpha)>-1$ and $E_{\alpha, \beta}(z)$ is the Mittag-Leffler function, defined by (6.2)

\section{REFERENCES}

[1] E.Orsingher, and L.Beghin. Time- fractional telegraph equations and telegraph processes with brownian time, Probability .Theory Relat. Fields, Vol.128, 141-160, 2004.

[2] R.K.Saxena.,A.M.Mathai , and H.J.Haubold. Reactiondiffusion systems connected with nonlinear waves, Astrophysics and Space Science.Vol. 305 ,297-303(2006).
[3] J.D.Murray . Mathematical Biology, New York,1989.

[4] M.C.Cross, and P.C.Hohenberg.Pattern formation outside of equilibrium,Rev. Mod. Phys. Vol. 65 , 851-912, 1993.

[5] Turing, A. The chemical basis of morphogenesis, Trans. Roy. Soc.. London Vol. B 237, 37 ,1952.

[6] B.I. Henry, and S.L.Wearne. Fractional reaction -diffusion , Physica A Vol. 276, 448-455,2000.

[7] D.E,Strier., D.H.Zanette, and H.S.Wio. Wave fronts in a bistable reaction - duffusion system with density- dependent diffusivity, Physica A, Vol.226, 310-323 (1995). 
[8] R. Gorenflo., A. Iskenderov, and Yu. Luchko. Mappings between solutions of fractional diffusion -wave equations, Fract. Calc.Appl. Anal., Vol. 3 , No.1, 75-86 (2000).

[9] R,Hilfer.(Editor). Applications of Fractional Calculus in Physics, World Scientific, Singapore, 2000.

[10] F.Mainardi, F., Yu.Luchko, and G. Pagnini . The fundamental solution of space-time fractional diffusion equation, Fract. Calc. Appl. Anal., Vol. 4, 153-192, 2001.

[11] F.Mainrdi., G.Pagnini, and R.K. Saxena. Fox H-functions in fractional diffusion,, J. Comput. Appl. Math .,Vol.178, 321-331, 2005

[12] A.I.Saichev, A.I.and G.M.Zaslavsky. Fractional kinetic equations: Solution and Applications, Chaos, Vol. 7 , No.4, 753-764, 1997.

[13] R.Metzler, and J.Klafter. The random walk's guide to anomalous .diffusion : a fractional dynamics approach , Physics reports ,Vol. 339, 1- 77, 2000.

[14] Metzler, R. and Nonnenmacher, T.F.: 2002, Space-and time-fractional diffusion and wave equations, Fokker- Planck equations, and physical motivation, Chemical Physics, 284 , $67-90$.

[15] V.V. Anh, and N.N.Leonenko. Spectral analysis of fractional kinetic equations with random data, J. Statistical Phys. Vol.104, Nos. 5/6,1349-1387, 2001

[16] H.J. Haubold., A,M. Mathai,and , R.K.Saxena. Solutions of fractional reaction-diffusion equations in terms of the H-function, Bull. Astr. Soc., India, 35, 681-689, 2007.

[17] I .Podlubny. Fractional Differential equations, Academic Press, San Diego,1999.

[18] A.A.Kilbas., H.M. Srivastava, and J.J.Trujillo. Theory and Applications of Fractional Differential Equations,, Elsevier, Amsterdam, 2006.

[19] R.K.Saxena. , A.M.Mathai and H.J.Haubold . On fractional kinetic Equations, Astrophysics and Space Science, Vol. 282, $281-287,2002$.

[20] R.K.Saxena.,A.M. Mathai, and H.J.Haubold . On generalized fractional kinetic equations, Physica A , Vol.344, 657-664.2004.

[21] R.K.Saxena., A.M.Mathai, and H.J.Haubold. Unified fractional kinetic equation and a fractional diffusion equation, Astrophysics and Space Science. Vol.290, No. 3\& 4, 299-310, 2004a.

[22] R.K.Saxena. , A.M.Mathai, and H.J. Haubold, H.J. Astrophysical thermonuclear functions for Boltzmann-Gibbs statistics and Tsallis statistics, Physica A 344,649-656. 2004b.

[23] R.K.Saxena., A.M.Mathai, and H.J.Haubold. Fractional reaction- diffusion equations, Astrophysics and Space
Science, Vol. 305. 297-303, 2006a.

[24] R.K.Saxena .,A.M. Mathai, and H.J.Haubold . Solution of generalized reaction-diffusion equations, Astrophysics and Space Science, Vol..305, 305-313, $2006 \mathrm{~b}$.

[25] I.M.Gelfand and G.F.Shilov. Generalized Functions, Vol. 1, Academic Press, London, 1964.

[26] Yu. Brychkov., and A.P.Prudnikov. Integral Transforms of Generalized Functions, Gordon and Breach Publishers,New York,,1989.

[27] G.M Mittag- Leffler. Sur la nouvelle function $E_{\alpha}(x)$. C.R. Acad. Sci.,Paris, Vol. 137, 554-558, 1903

[28] Wiman. Über den Fundamental satz in der Theorie der Functionen $\mathrm{E}^{\alpha}$ (x), Acta Math.Vol. 29, 191 -201, 1905.

[29] Erdélyi., W,Magnus.,F, Oberhettinger, and F.G.Tricomi. Higher Transcendental Functions , Vol. 3, McGraw Hill ,New York, Toronto, 1955.

[30] M.M.Dzherbashyan, M.M. Integral Transforms and Representation of Functions in Complex Domain (in Russian ), Nauka, Moscow1966.

[31] M.M.Dzherbashyan. Harmonic Analysis and Boundary Value Problems in the Complex Domain, Birkhaüser -Verlag ,Basel , and London, 1993.

[32] T.R.Prabhakar. A singular integral equation with generalized Mittag-Leffler function in the kernel, Yokohama Math. J, Vol. $19,7-15,1971$

[33] A.A.Kilbas., M. Saigo, and R.K.Saxena. Solution of Volterra integro-differential equations with generalized Mittag-Leffler functions in the kernels, J. Integral Equations, Vol. 14,No. 4,377-396, 2002.

[34] A.A.Kilbas.,M. Saigo, and R.K.Saxena. Generalized Mittag-Leffler Function and Generalized Fractional Calculus, Integral Transforms and Special Functions, Vol. 15, No. 1, 31-49, 2004.

[35] A,M,Mathai, R,K,Saxena, and H.J.Haubold, The H-function : Theory and Applications, Springer, New York, Dordrecht, Heidelberg, London, 2010.

[36] M.Caputo. 1969, Elasticita e Dissipazione, Zanichelli,Bologna, 1969.

[37] S.G.Samko.,A.A. Kilbas, and O.I.Marichev. Fractional Integrals and Derivatives, Theory and Applications, Gordon and Breach Publishers, New York, 1993.

[38] G.Doetsch. Anleitung zum Praktischen Gebrauch der Laplace transformation, Oldenbourg, Munich, 1956.

[39] M.Abramowitz and I.A.Stegun. Handbook of Mathematical functions with Formulas, Graphs, and Mathematical Tables, Applied Mathematics Series 55, 7th Printing, National Bureau of Standards, Washington , D.C.,1968. 